cervix, Okabe and co-workers (Biochemistry, 7,79 ; 1968) have found, like many other workers working with many other kinds of tumours, that there is a preponderance of isozymes containing $M$ sub-units in the malignant tissue from tumours. They found, for example, that the benign uterine tumour had an isozyme pattern very similar to the pattern in normal human uterus. They isolated the isozymes from the tissues and found that those from the malignant tissue showed a higher rate of reaction with nicotinamide adenine dinucleotide analogues than the other types.

An enhanced production of lactic acid from pyruvic acid (brought about by $\mathrm{LDH}$ ) is the outstanding biochemical difference between malignant cells and normal cells. Indeed, when Warburg first discovered the high rate of glycolysis in tumours, he suggested that this was the cause of malignant growth. Opinion has recently tended to swing away from this theory, but there is now some evidence that the change in LDH isozymes may be one of the earliest alterations when a tissue becomes malignant and that this change in $\mathrm{LDH}$ may influence the rate of glycolysis.

In 1966, Latner, Turner and Way (Lancet, ii, 814; 1966) found that in specimens of pre-invasive carcinoma of the cervix obtained at operation, the amount of change in the LDH pattern seems to be correlated with the amount of tumour tissue. In some cases, however, the LDH pattern was changed even though there was no evidence of carcinoma when sections were examined. All the specimens must have contained some tumour cells, for cancer had been diagnosed from cervical smears. The LDH isozymes were changed before any change in the activity of lactate, glucose 6-phosphate or 6-phosphogluconate dehydrogenases was measurable.

Langvad (Inter. $J$. Cancer, 3, 17; 1968) has now measured the ratio of $\mathrm{LDH}$ isozymes with $M_{3} H_{1}$ and $M_{1} H_{3}$ constitutions in samples from the tumour and from apparently normal colon of operation specimens from thirty-six patients with carcinoma of the colon. Each tissue sample was halved longitudinally, one half being used for the preparation of $\mathbf{L D H}$ isozymes and the other prepared for histological examination. The mean ratio for samples containing tumour tissue was $1 \cdot 5-1 \cdot 6$, while the mean ratio for samples with no tumour tissue was $0 \cdot 7-0 \cdot 8$. Usually the ratio decreased with distance from the tumour, but some specimens had local areas where the ratio rose considerably above 0.8 and approached the ratio found in the tumours. These patches where the ratio increased were often at considerable distances from the edge of the tumour. It is not unusual to find more than one primary tumour in patients with carcinoma of the colon, so these areas where the LDH ratio changes towards the tumour ratio may well be areas which will eventually become malignant. The change in $\mathrm{LDH}$ isozymes seems to occur before the tissue looks malignant, and may be one of the earliest detectable changes in a tissne which is developing a tumour.

\section{Phage RNA Replication}

\section{from our Cell Biology Correspondent}

For several years, there has been great interest in the mechanism by which the single-stranded RNA molecules of coliphage are synthesized. Weissmann's group (Feix, Pollet and Weissmann, Proc. US Nat. Acad. Sci., 59, 145; 1968) has now demonstrated that the minus strand R.NA molecules of $Q \beta$ coliphage, which are inherently non-infectious, can serve as templates for the in vitro synthesis of $Q \beta$ plus strands. Last year Weissmann proposed that the minus strand - not a duplex molecule with plus and minus strands hydrogen bonded together-is the template for synthesis of progeny plus strands during the replication of the single-stranded RNA genome of $Q \beta$ (see Nature, 214, 1287; 1967). Two obscrvations supported this idea. First, some of the minus strands made in vivo in $Q \beta$ infected $E$. coli occur in a complex with molecules of replicase and with plus strands but are not hydrogen bonded to the plus strands. Secondly, neither of the two species of double-stranded RNA moleculesreplicative form and replicative intermediate-which can be extracted with detergent and phenol from $Q \beta$ infected $E$. coli serves as a template for $Q \beta$ replicase in vitro unless it has been denatured by heat. The only way to test this hypothesis, however, was to isolate minus strands and investigate their template properties.

In November last year (see Nature, 216, 639; 1967) Weissmann's group reported the isolation of pure $Q \beta$ minus strands. Now they have found that the minus strands do indeed direct a rapid synthesis of $Q \beta$ plus strands. In the presence of the four nucleotide triphosphates, $Q \beta$ minus strands promote rapid synthesis of RNA plus strands by $Q \beta$ replicase. In fifteen minutes the amount of RNA synthesized is three times the input of RNA, but with $Q \beta$ plus strands as template, less RNA and fewer infectious units are made. Furthermore, the enzyme is saturated by a much lower concentration of plus strands than minus strands and, at high template concentrations, incorporation into RNA is about seven times greater with minus strand than with plus strand templates. Feix et al. calculate that with minus strands as templates, a plus strand is made in about 100 seconds, which means that about 30 phosphodiester bonds are formed per second. These results are compatible with the assumption that the replicase preparation contains a small number of binding sites specific for plus strands and a larger number of binding sites specific for minus strands. Whether these two sites are on different sub-units of one protein or on two different proteins remains to be seen.

Spiegelman's group, unconvinced by the evidence obtained by others for the existence of a minus strand and its role in replication, has been doing experiments to convince itself. First, the group has satisfied itself that a minus strand exists and, in its latest contribution to the Proc. US Nat. Acad. Sci. (59, 141; 1968), Pace, Bishop and Spiegelman conclude that replicative form and intermediate, which they had chosen to call Hofschneider and Franklin structures, are in fact involved in $Q \beta$ replication and deserve their original names.

Also in the current Proc. US Nat. Acad. Sci. (59, 185 ; 1968), Nayak and Baluda describe the isolation, by phenol extraction, of replicative intermediate RNA molecules from chick embryo cells infected with equine influenza virus. Because replicative intermediates have also been found in cells infected with polio virus, it appears that all single-stranded viral RNA molecules are replicated by the same basic mechanism. 